\title{
ON THE DIMENSION OF THE JACQUET MODULE OF A CERTAIN INDUCED REPRESENTATION
}

\author{
William Banks, Daniel Bump, and Daniel Lieman \\ Dedicated to Jim Huckaba, on the occassion of his retirement
}

The Fourier coefficients of certain metaplectic Eisenstein series contain $L$-series of arithmetic interest. This fact has been repeatedly exploited by various authors in order to obtain analytic information about these $L$-series. Bump and Hoffstein $[\mathrm{H}]$ conjectured that the Hecke $L$-series of the $n$-th order residue symbol would be contained in the Fourier coefficients of an Eisenstein series on the $n$-cover of $G L(n)$ induced up from a theta function on the $n$-cover of $G L(n-1)$. Some evidence for this conjecture was provided in $[\mathrm{H}]$, and a representation theoretic explanation for its plausibility was given in [BL]. Recently, Suzuki [S] proved the conjecture in the function field case, and he carried out many preliminary steps towards the proof of the conjecture in the number field case. Using very different techniques, the authors of this paper recently proved a generalization of the (slightly corrected version of the) Bump-Hoffstein conjecture over an arbitrary global field [BBL].

In the course of the proof in [BBL], the authors considered various means of attack on the problem. One of these was the method of Casselman and Shalika, which required us to bound the size of the

This work was partially supported by NSF grants DMS-9622819 (Bump) and DMS-9700542 (Lieman). The third author would like to thank MSRI, Berkeley, for its hospitality during part of the time this research was conducted. The first and third authors would like to thank Macquarie University for its gracious hospitality during the preliminary preparation of this note. We would also like to thank the Chicago Cubs for finally winning enough baseball games for Jim to consider it worthwhile to retire and go watch spring training.

Typeset by $\mathcal{A M S}_{\mathcal{M}}-\mathrm{T}_{\mathrm{E}} \mathrm{X}$ 
Jacquet module $J V$ of a certain induced representation. Although this work was not needed in the final version of our paper, the computation of $\operatorname{dim}(J V)$ is both amusing and enlightening, and we present it here as a pleasant result in its own right. The basic reference for this material is $[\mathrm{KP}]$.

For $n \geq 3$, the setup is as follows. Let $F$ be a nonarchimedean local field such that the group of $n$-th roots of unity in $F^{\times}$has cardinality $n$. Let $\Theta$ denote the theta representation on the $n$-fold (metaplectic) cover of $G L(n-1, F)$, let $G$ denote the $n$-fold cover of $G L(n, F)$, and let $\delta_{P}$ be the modular character of the $(n-1,1)$-parabolic $P$ of $G$. Once and for all, we fix an element $s \in \mathbb{C}$. Let $(\rho, V)$ be the (normalized) induced representation $\operatorname{Ind}_{P}^{G}\left(\Theta \otimes \delta_{P}^{s}\right)$, and let $J V$ be the Jacquet module defined by

$$
J V=V /\langle\rho(n) v-v \mid v \in V, n \in N\rangle,
$$

where $N$ is the canonical lift of the standard maximal unipotent subgroup of $G L(n, F)$. Note that $J V$ is an admissible $T$-module, where $T$ denotes the metaplectic preimage in $G$ of the diagonal matrices. Our goal in this paper is to show that $\operatorname{dim}(J V)=n$ (a priori, one only knows that $\operatorname{dim}(J V) \leq n !)$.

To illustrate the ideas, we consider first the case $n=3$. Let $\omega$ be the character of $T$ given by

$$
\left(\begin{array}{ccc}
y_{1} & & \\
& y_{2} & \\
& & y_{3}
\end{array}\right) \mapsto\left|y_{1}\right|^{s+1 / 6}\left|y_{2}\right|^{s-1 / 6}\left|y_{3}\right|^{-2 s} .
$$

Let $\operatorname{Ind}_{B}^{G}(\omega)$ be the representation of $G$ parabolically induced from $\omega$ (normalized induction) as explained in Kazhdan and Patterson [KP]. Then $V$ is the unique subrepresentation of $\operatorname{Ind}_{B}^{G}\left({ }^{s_{1}} \omega\right)$, where we have written $s_{1}$ for the matrix

$$
\left(\begin{array}{lll} 
& 1 & \\
1 & & \\
& & 1
\end{array}\right)
$$

(more precisely, $s_{1}$ is the canonical preimage of this matrix in $G$ ). Note that $V$ is also the unique quotient representation of $\operatorname{Ind}_{B}^{G}(\omega)$. For 
every element $w$ of the Weyl group, let ${ }^{w} \omega$ be the character defined by ${ }^{w} \omega(t)=\omega\left(w^{-1} t w\right)$. It is known that $\operatorname{dim}\left(\operatorname{Hom}_{T}\left(J V,{ }^{w} \omega\right)\right) \leq 1$. We will prove that for precisely 3 elements $w, \operatorname{dim}\left(\operatorname{Hom}_{T}\left(J V,{ }^{w} \omega\right)\right)=1$; the assertion that $\operatorname{dim}(J V)=3$ follows immediately.

Indeed, suppose that $\sigma$ is a nonzero element of $\operatorname{Hom}_{T}\left(J V,{ }^{w} \omega\right)$. By Frobenius reciprocity, $\operatorname{Hom}_{T}\left(J V,{ }^{w} \omega\right) \cong \operatorname{Hom}_{G}\left(V, \operatorname{Ind}_{B}^{G}\left({ }^{w} \omega\right)\right)$, so we may regard $\sigma$ as a nonzero element of $\operatorname{Hom}_{G}\left(V, \operatorname{Ind}_{B}^{G}\left({ }^{w} \omega\right)\right)$. Now consider the composition

$$
\operatorname{Ind}_{B}^{G}\left({ }^{s_{1}} \omega\right) \stackrel{T_{s_{1}}}{\longrightarrow} \operatorname{Ind}_{B}^{G}(\omega) \stackrel{T_{s_{1}}}{\longrightarrow} V \stackrel{\sigma}{\longrightarrow} \operatorname{Ind}_{B}^{G}\left({ }^{w} \omega\right) .
$$

By [KP, 1.2.2], there exists a constant $c \neq 0$ so that $\sigma \circ T_{s_{1}}=c T_{w}$. Using the definition of $\omega$, it follows from [KP, 1.2.6] that $T_{s_{1}} \circ T_{s_{1}}=0$. Thus, $0=\sigma \circ T_{s_{1}} \circ T_{s_{1}}=c T_{w} \circ T_{s_{1}}$. This yields a contradiction unless $\ell\left(w s_{1}\right)=\ell(w)-1$, since $T_{w} \circ T_{s_{1}}=T_{w s_{1}} \neq 0$ if $\ell\left(w s_{1}\right)=\ell(w)+1$ by [KP, 1.2.3].

Conversely, if $\ell\left(w s_{1}\right)=\ell(w)-1$, then the composition $T_{w s_{1}} \circ T_{s_{1}}$ from $\operatorname{Ind}_{B}^{G}(\omega)$ to $\operatorname{Ind}_{B}^{G}\left({ }^{w} \omega\right)$ is a nonzero multiple of $T_{w}$. By restricting $T_{w s_{1}}$ to $V=\operatorname{Im}\left(T_{s_{1}}\right)$, one obtains a nonzero element $\sigma=\left.T_{w s_{1}}\right|_{V}$ in $\operatorname{Hom}_{G}\left(V, \operatorname{Ind}_{B}^{G}\left({ }^{w} \omega\right)\right) \cong \operatorname{Hom}_{T}\left(J V,{ }^{w} \omega\right)$. Consequently, we've proved (for $n=3$ )

\section{PROPOSITION.}

$$
\operatorname{dim}\left(\operatorname{Hom}_{T}\left(J V,{ }^{w} \omega\right)\right)=1 \quad \Longleftrightarrow \quad \ell\left(w s_{1}\right)=\ell(w)-1 .
$$

For arbitrary $n \geq 3$, the proof that $\operatorname{dim}(J V)=n$ is very similar. We include it here for the sake of completeness. Let $w$ now denote the matrix

$$
\left(\begin{array}{llll} 
& & 1 & \\
& . & & \\
1 & & & \\
& & & 1
\end{array}\right) .
$$

There exist $n-2$ distinct simple reflections $s_{i}$ such that $T_{w} \circ T_{s_{i}}=0$. Let $\omega$ now be the character such that $V$ is the unique subrepresentation of $\operatorname{Ind}_{B}^{G}\left({ }^{w} \omega\right)$. Again, we choose a Weyl group element $w^{\prime}$ and an element $\sigma \neq 0$ in

$$
\operatorname{Hom}_{T}\left(J V,{ }^{w^{\prime}} \omega\right) \cong \operatorname{Hom}_{G}\left(V, \operatorname{Ind}_{B}^{G}\left(w^{\prime} \omega\right)\right) .
$$


Consider the picture

$$
\operatorname{Ind}_{B}^{G}\left({ }^{s_{i}} \omega\right) \stackrel{T_{s_{i}}}{\longrightarrow} \underbrace{\operatorname{Ind}_{B}^{G}(\omega) \stackrel{T_{w}}{\longrightarrow} V \stackrel{\sigma}{\longrightarrow} \operatorname{Ind}_{B}^{G}\left(w^{\prime} \omega\right)}_{c T_{w^{\prime}}} .
$$

By $[\mathrm{KP}, 1.2 .2,1.2 .3$ and 1.2.6], as before, there exists a constant $c \neq 0$ with $\sigma \circ T_{w}=c T_{w^{\prime}}$. On the other hand, $T_{w} \circ T_{s_{i}}=0$, and so $0=\sigma \circ T_{w} \circ T_{s_{i}}=c T_{w^{\prime}} \circ T_{s_{i}}$. This is a contradiction unless

$$
\ell\left(w^{\prime} s_{i}\right)=\ell\left(w^{\prime}\right)-1, \quad i=1,2, \ldots, n-2 .
$$

Conversely, if $\ell\left(w^{\prime} s_{i}\right)=\ell\left(w^{\prime}\right)-1$ for every $i=1, \ldots, n-2$, it follows that $\ell\left(w^{\prime}\right)=\ell\left(w^{\prime} w^{-1}\right)+\ell(w)$, hence the composition $T_{w^{\prime} w^{-1}} \circ T_{w}$ from $\operatorname{Ind}_{B}^{G}(\omega)$ to $\operatorname{Ind}_{B}^{G}\left({ }^{w^{\prime}} \omega\right)$ is a nonzero multiple of $T_{w^{\prime}}$. By restricting $T_{w^{\prime} w^{-1}}$ to $V=\operatorname{Im}\left(T_{w}\right)$, we obtain a nonzero element $\sigma=\left.T_{w^{\prime} w^{-1}}\right|_{V} \in$ $\operatorname{Hom}_{G}\left(V, \operatorname{Ind}_{B}^{G}\left(w^{\prime} \omega\right)\right) \cong \operatorname{Hom}_{T}\left(J V,{ }^{w^{\prime}} \omega\right)$. Thus, we've proved

\section{PROPOSITION.}

$\operatorname{dim}\left(\operatorname{Hom}_{T}\left(J V,{ }^{w^{\prime}} \omega\right)\right)=1 \Longleftrightarrow \ell\left(w^{\prime} s_{i}\right)=\ell\left(w^{\prime}\right)-1$ for $1 \leq i \leq n-2$.

To see what this means for $w^{\prime}$, we note that condition (1) means $w^{\prime} \alpha_{i}<0$ for all $1 \leq i \leq n-2$ where $\alpha_{i}$ ranges over the first $(n-2)$ positive simple roots. In other words, if $w^{\prime} \in S_{n}$, we require that $w^{\prime}(i) \geq w^{\prime}(j)$ whenever $1 \leq i \leq j \leq n-2$. For example, the surviving elements of the Weyl group in $G L(3)$ are

$$
\left(\begin{array}{lll}
1 & 1 & \\
& & \\
& & 1
\end{array}\right), \quad\left(\begin{array}{lll} 
& 1 & \\
& & 1 \\
1 & &
\end{array}\right), \quad\left(\begin{array}{lll} 
& & 1 \\
& 1 & \\
1 & &
\end{array}\right),
$$

while the surviving elements of the Weyl group in $G L(4)$ are

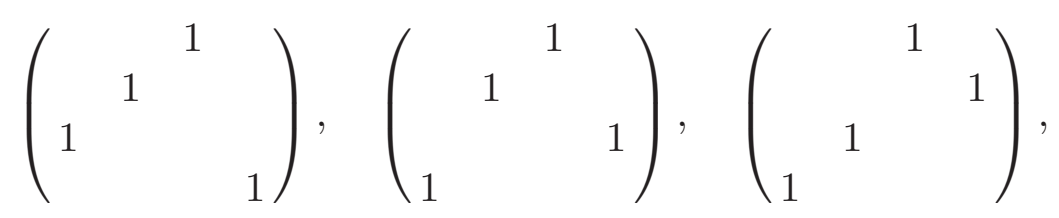




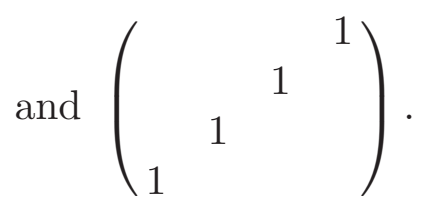

In general, the Weyl group in $G L(n)$ will have precisely $n$ surviving elements, and the assertion that $\operatorname{dim}(J V)=n$ then follows immediately from the proposition.

\section{REFERENCES}

[BBL] W. Banks, D. Bump and D. Lieman, On the Whittaker-Fourier coefficients of metaplectic Eisenstein series, in preparation.

[BL] D. Bump and D. Lieman, Uniqueness of Whittaker functionals on the metaplectic group, Duke Math. J. 76, 731-739.

$[\mathrm{H}] \mathrm{J} . \mathrm{Hoffstein}$, Eisenstein series and theta functions on the metaplectic group, Theta functions: from the classical to the modern, CRM Proc. Lecture Notes, Amer. Math. Soc. 1 (1993), 65-104.

[KP] D. Kazhdan and S. Patterson, Metaplectic forms, Publ. Math. IHES 59.

[S] T. Suzuki, Metaplectic Eisenstein series and the Bump-Hoffstein conjecture, Duke Math. J. 90, 577-630.

Department of Mathematics, University of Missouri, Columbia, MO 65211

E-mail address: bbanks@math.missouri.edu

Department of Mathematics, Stanford University, Palo Alto, CA 94305

E-mail address: bump@math.stanford.edu

Department of Mathematics, University of Missouri, Columbia, MO 65211

E-mail address: lieman@math.missouri.edu 